\title{
Actualización del catálogo florístico de Sierra Morena oriental (centro- sur de la Península Ibérica, España)
}

\section{Carlos Fernández García-Rojo ${ }^{1}$ \& Carlos Salazar Mendías²}

'Instituto de Enseñanza Secundaria "Francisco Nieva" Avenida de los Estudiantes, 99. E-13300- Valdepeñas (Ciudad Real, España).

${ }^{2}$ Dpto. Biología Animal, Biología Vegetal y Ecología. Facultad de Ciencias Experimentales. Universidad de Jaén. E-23071- Jaén (España).

\author{
Correspondencia \\ C. Salazar Mendías \\ e-mail: csalazar@ujaen.es \\ Recibido: 14 diciembre 2018 \\ Aceptado: 11 septiembre 2019 \\ Publicado on-line: 2 octubre 2019 \\ Editado por: B. Cabezudo
}

\begin{abstract}
Update of the floristic catalog of Eastern Sierra Morena (south-central Iberian Peninsula, Spain)
\end{abstract} Palabras clave: Corología, flora vascular, Ciudad Real, Jaén, Albacete,
Castilla-La Mancha, Andalucía.

Key words: Chorology, vascular flora, Ciudad Real, Jaén, Albacete, Castile-La Mancha, Andalusia.
Sierra Morena oriental comprende las sierras del este de la cordillera Mariánica, desde las sierras de Andújar (norte de la provincia de Jaén) y Madrona (sur de Ciudad Real) hasta alcanzar puntualmente la sierra de Relumbrar (Albacete). Por tanto, su territorio se extiende por las comunidades autónomas de Andalucía y CastillaLa Mancha.

Siguiendo el criterio biogeográfico de RivasMartínez (2007) el área pertenece al distrito Marianense Oriental, subsector Marianense, sector Mariánico-Monchiquense, subprovincia Luso-Extremadurense. Sierra Morena oriental está delimitada aproximadamente por las coordenadas geográficas de latitud: $38.65 \mathrm{~N}$ a $38 \mathrm{~N}$ y longitud: -2.70 O a -4.300 .

El catálogo florístico de Sierra Morena oriental recientemente publicado (Fernández García-Rojo, 2015) contenía 838 taxones herborizados en las últimas tres décadas. Posteriores revisiones del material de herbario han permitido corregir varias especies de los géneros Coincya, Gagea, Milium y Silene, lo que junto a la adición de las herborizaciones realizadas entre 2016 y 2018 eleva a 842 los taxones hallados en esta zona. Asimismo, se han herborizado en nuevas localidades otras 2 especies previamente conocidas en el territorio.

Los pliegos se han depositado en el herbario de la Universidad de Jaén (JAEN). Hemos comprobado que, a pesar de que sus materiales han sido generalmente revisados para la edición de las recientes floras de nuestro país, ciertas especies han pasado desapercibidas, suponiendo primeras o segundas citas para las provincias de Albacete, Ciudad Real y Jaén, y destacan algunas novedades florísticas y/o únicas herborizaciones para las floras de Andalucía y Castilla-La Mancha.

Para comprobar la distribución de los 13 taxones que se exponen a continuación de forma alfabética, se ha consultado la información disponible en las bases de datos de Anthos (2019) y GBIF (2019), además de la corología señalada en Flora iberica (Castroviejo, 1986-2019) y Flora Vascular de Andalucía Oriental (Blanca et al., 2011), así como diversos catálogos y artículos botánicos que se citan debidamente en el texto.

Aster aragonensis Asso, Syn. Stirp. Aragon.: 117 tab. VIII fig. 2, 121 (1779)

(= Galatella aragonensis (Asso) Nees, Gen. Sp. Aster.: 167 (1832))

En un principio identificada como Aster sedifolius L. subsp. sedifolius (Fernández García-Rojo \& Fernández López, 1992: 21) y posteriormente corregida como Aster aragonensis Asso.

Su presencia en Sierra Morena oriental ha sido ampliamente documentada en la provincia de Ciudad Real (García Río, 1998; Martín Blanco \& Carrasco, 2005) pero fue citada por primera vez en la provincia de Jaén y Andalucía en el catálogo (Fernández García-Rojo, 2015: 20).

Sin embargo, no fue incluida en Flora Vascular de Andalucía Oriental (Blanca, 2011) a pesar de que el pliego herborizado en territorio giennense data de 1990. Según se ha podido comprobar, por 
alguna razón que desconocemos, este material no fue revisado durante la elaboración de la obra.

Asimismo, su presencia en la provincia de Jaén no se indica en la reciente publicación del género en Flora iberica (Aedo, 2019).

Jaén:SantaElena, las Correderas, 30SVH5350, 980 m, brezal, 15/9/1990, C. Fernández GarcíaRojo (JAEN 901261).

Carex reuteriana Boiss. subsp. reuteriana, Pugill. Pl. Afr. Bor. Hispan.: 116 (1852)

Aportamos una herborización en el arroyo Ballesteros, donde ya se había observado sin colectarlo (Fernández García-Rojo, 2015: 32, sub C. elata subsp. reuteriana (Boiss.) Luceño \& Aedo).

Ciudad Real: Viso del Marqués, arroyo de Ballesteros, 30SVH3555, UTM: $x=0435562$, y $=4255480,863 \mathrm{~m}$, macollar en lecho del arroyo, 2/5/2018, C. Fernández García-Rojo (JAEN 184002).

Coincya rupestris subsp. leptocarpa (Gonz.Albo) Leadlay, Bot. J. Linn. Soc. 102(4): 364 (1990)

Se ha revisado el material del género Coincya, comprobándose que los pliegos JAEN 114037, 114055 y 114066 corresponden a C. rupestris subsp. leptocarpa, y no a Coincya longirostra (Boiss.) Greuter \& Burdet, como anteriormente se indicó (Fernández García-Rojo, 2015: 41). Por tanto, se trata de nuevas localidades de este taxón en el área de estudio.

Coincya rupestris Porta \& Rigo ex Rouy, Naturaliste 2, 13(111): 248 (1891) subsp. rupestris

La revisión minuciosa del material del género Coincya nos lleva a considerar que el pliego JAEN 144014 corresponde a $C$. rupestris subsp. rupestris, y no a Coincya longirostra (Boiss.) Greuter \& Burdet como inicialmente se citaba (Fernández GarcíaRojo, 2015: 41). Se trata de una nueva adición al catálogo.

Gagea foliosa subsp. ellyptica A. Terracc., Bol. Soc. Aragonesa Ci. Nat. 4: 238 (1905)

En el catálogo de Sierra Morena oriental se señalaba la existencia de cuatro taxones del género Gagea, pero una revisión minuciosa del material siguiendo el criterio de López González (2013) ha puesto de manifiesto que en todos los casos se trata de Gagea foliosa subsp. ellyptica. Este es el caso del material contenido en los pliegos JAEN 704002 y 704006, identificados como G. bohemica (Zauschn.) Schult. \& Schult. fil. (Fernández García-Rojo, 2015: 63); JAEN 901126 y 916002 , identificados como G. foliosa subsp. durieui (Parl.) G. López (Fernández GarcíaRojo, 2015: 63), y JAEN 114007, 114016, 114018, 124002,694019 y 924005 identificados como $G$. lacaitae A. Terracc. (Fernández García-Rojo, 2015: 63). Por tanto, estos tres taxones deben eliminarse del catálogo original.

De especial interés es la detección de Gagea foliosa subsp. ellyptica en la provincia de Albacete, donde no la indica López González (2013). Esto hace pensar que en concreto el pliego JAEN 124002 no fue revisado en su momento, por haberse colectado en el año 2012, cuando ya se estaba editando el género.

Los registros de G. foliosa en la provincia de Albacete que se pueden consultar en Anthos, o bien corresponden a Gagea foliosa subsp. durieui (sub G. iberica A. Terracc.) (Sánchez-Gómez \& Alcaraz, 1993) o no concretan la subespecie de la que se trata (Montserrat, 1981). Los datos de GBIF señalan la presencia de $G$. foliosa en Albacete con dos pliegos herborizados en el Calar del Mundo (ABH 53818 y SEV 21771). Sin embargo, se ha comprobado que realmente deben corresponder a G. lacaitae (Martínez Azorín, com. pers.).

Por tanto, concluimos que esta es la primera cita publicada de $G$. foliosa subsp. ellyptica en la provincia de Albacete.

Albacete: Villapalacios, alrededores Hoya Quemada, 30SWH2470, $\mathrm{x}=0524025, \mathrm{y}=$ 4270976, $1126 \mathrm{~m}$, clastos cuarcíticos con matorral de jara pringosa, cantueso, labiérnago y enebro, 16/3/2012, C. Fernández García-Rojo (JAEN 124002).

\section{Milium effusum L., Sp. Pl.: 61 (1753)}

Tras la revisión del pliego JAEN 934070 atribuido a Milium vernale subsp. montianum (Parl.) K. Richt. (Fernández García-Rojo, 2015: 94), concluimos que se trata de $M$. effusum.

Por tanto, ha de descartarse la presencia en el área de $M$. vernale subsp. montianum.

Neoschischkinia elegans (Loisel.) Tzvelev, Bot. Journ., URSS 53: 309, in obs. (1968)

(= Agrostis tenerrima Trin., Gram. Unifl. Sesquifl. 205 (1824))

Nueva adición al catálogo de Sierra Morena oriental.

Ciudad Real: San Lorenzo de Calatrava, collado de la Rebollera, 30SVH2852, UTM: $\mathrm{x}=$ 0428144, y = 4252628, $1030 \mathrm{~m}$, pastizal con Pinus pinaster, 18/6/2016, C. Fernández García-Rojo (JAEN 164002).

Sambucus nigra L., Sp. Pl.: 269 (1753) subsp. nigra 
Nueva adición al catálogo de Sierra Morena oriental.

Ciudad Real: Viso del Marqués, los Chorros, 30SVH3959, UTM: $x=0439412, y=4259713,846$ m, aliseda, 9/6/2016, C. Fernández García-Rojo (JAEN 164001).

Silene mariana Pau, Mem. Real Soc. Esp. Hist. Nat., Tomo Extraord.: 291 (1921)

Inicialmente considerada en el catálogo como Silene psammitis Link ex Sprengel subsp. psammitis (Fernández García-Rojo, 1998: 270; Fernández García-Rojo, 2015: 142) a partir de un pliego herborizado en 1990. Este material ha sido revisado recientemente, revelando que se trata de $S$. mariana, endemismo amenazado del sur peninsular.

La presencia de esta especie en la provincia de Ciudad Real es referida por Talavera (1990), sin duda basada en el pliego SEV 219526 (Valle de Alcudia, entre Bienvenida y El Alamillo). Además hay una cita poco concreta recogida por Domínguez Lozano et al. (1994: 200) a partir de un comentario personal del propio Talavera, que indica su presencia en un lugar indeterminado entre Chillón y Peñalsordo, que los autores circunscriben a la cuadrícula UH39 (claramente alejada de Sierra Morena oriental).

La presencia de este taxón en Sierra Morena oriental está mejor documentada en la provincia de Jaén donde ha sido herborizada en distintas localidades de los parques naturales de la Sierra de Andújar y Despeñaperros (Fernández López et al., 2006).

Por tanto, se trata de la segunda cita refrendada con material de herbario en Ciudad Real, y por ende en Castilla-La Mancha. Asimismo ha de contemplarse como una nueva adición al catálogo que nos ocupa.

Este dato corológico es de especial relevancia, dado que $S$. mariana se encuentra protegida por la legislación autonómica de Castilla-La Mancha, y se halla incluida en la Directiva de Hábitats de la UE. El estado actual de las poblaciones de esta especie en dicha región no parecen ser conocidas (Salazar et al., 2019).

Ciudad Real: Viso del Marqués, Charco Batán, 30SVH4351, $700 \mathrm{~m}$, jaral aclarado en terraza arenosa, 3/5/1990, C. Fernández García-Rojo (JAEN 901180). (Rev. C. Díaz de la Guardia, 2/2006; C. Salazar, 2/2017).

Simethis mattiazzii (Vand.) Sacc., Atti Mem. Reale Accad. Sci. Lett. Arti Padova 16: 76 (1900) (= S. planifolia (Vand. ex L.) Gren. \& Godr., Fl.
France 3: 222 (1855)).

Aportamos la primera herborización de esta especie en Sierra Morena oriental, y más concretamente en la provincia de Ciudad Real donde fue citada por Rivas Goday et al. (1954, sub S. planifolia). Las citas fueron recogidas por Martín Blanco \& Carrasco (2005), García Río (2006) y Morales del Molino \& Mateo (2010), quienes indicaron que la especie no había sido nuevamente encontrada ni herborizada.

La primera herborización de esta especie en la comunidad autónoma de Castilla-La Mancha se debe a Morales del Molino \& Mateo (2010) que la localizan en la provincia de Guadalajara.

A pesar de las citas existentes, Martínez Ortega (2013) no la cita en la provincia de Ciudad Real, por lo que concluimos que el pliego que aportamos es sin duda la primera herborización en la provincia de Ciudad Real y la segunda herborización en Castilla-La Mancha. Asimismo ha de añadirse al catálogo que nos ocupa como la segunda cita comprobada en Sierra Morena oriental.

Ciudad Real: Viso del Marqués, sierra de la Estrella, 30SVH4750, UTM: $x=0447603, y=$ 4250386, $1259 \mathrm{~m}$, brezal de Erica australis con robles, 14/5/2017, C. Fernández García-Rojo (JAEN 174002).

Stipa bromoides (L.) Dörfler, Herb. Norm. 34: 129 (1897)

Nueva adición al catálogo de Sierra Morena oriental.

Ciudad Real: San Lorenzo de Calatrava, barranco de los Gavilanes, 30SVH2953, UTM: x = 0429677, y = 4253709, $869 \mathrm{~m}$, umbría quejigal, 30/6/2016, C. Fernández García-Rojo (JAEN 164003).

Tanacetum corymbosum (L.) Schultz Bip., Tanacet. 57 (1844)

Nueva adición al catálogo de Sierra Morena oriental.

Ciudad Real: San Lorenzo de Calatrava, barranco de los Gavilanes, 30SVH2953, UTM: x = 0429846, y = 4253720, $869 \mathrm{~m}$, umbría quejigal, 2/6/2017, C. Fernández García-Rojo (JAEN 174003).

Torilis japonica (Houtt.) DC., Prodr. 4: 219 (1830)

Algunos pliegos inicialmente identificados como Torilis purpurea (Ten.) Guss (Fernández García-Rojo, 1998) fueron posteriormente revisados como T. japonica (Fernández GarcíaRojo, 2015: 153). Este material no parece haber sido estudiado para la elaboración de la familia Apiaceae en Flora iberica (Jury, 2003) a pesar de 
tratarse de pliegos del año 1992. En dicha obra se indica la provincia de Ciudad Real entre paréntesis, por lo que no parece haber testimonios de herbario aparte del que aquí indicamos.

Se trata por tanto de la primera cita confirmada para la provincia de Ciudad Real.

Ciudad Real: Villanueva de San Carlos, arroyo Cabañas, 30SVH2672, $600 \mathrm{~m}$, herbazal en suelo húmedo de tamujal, 4/6/1992, C. Fernández GarcíaRojo (JAEN 924402). (Rev. S. Silvestre, 02/2005).

\section{Agradecimientos}

A los guardas, administradores y propietarios de las fincas visitadas durante tantos años. A Pablo Alcaraz Párraga por su colaboración en la búsqueda de pliegos en el Herbario JAEN. A Francisco Javier Salgueiro (herbario SEV). A Manuel Benito Crespo y Mario Martínez Azorín por suministrarnos la información del herbario $\mathrm{ABH}$. Y finalmente, a Carlos Fernández López que tanto ha hecho por el conocimiento botánico de la provincia de Jaén y de Andalucía oriental.

\section{Bibliografía}

Aedo, C. (2019). Aster L. In C. Benedí, A. Buira, E. Rico, M. B. Crespo, A. Quintanar \& C. Aedo (Eds.), Flora iberica vol. 16(3) (pp. 1981-1993). Madrid, España. Real Jardín Botánico, CSIC.

Anthos (2019). Sistema de información sobre las plantas de España (http://www.anthos.es/).

Blanca, G. (2011). Galatella Cass. In G. Blanca, B. Cabezudo, M. Cueto, C. Morales Torres \& C. Salazar (Eds.), Claves de la Flora vascular de Andalucía Oriental (pp. 1625-1626). Granada, España. Universidades de Granada, Almería, Jaén y Málaga.

Blanca, G., Cabezudo, B., Cueto, M., Morales Torres, C. \& Salazar, C. (Eds.) (2011). Claves de la Flora vascular de Andalucía Oriental. Granada, España. Universidades de Granada, Almería, Jaén y Málaga.

Castroviejo, S. (Coord.) (1986-2019). Flora iberica. Plantas vasculares de la Península Ibérica e Islas Baleares. Vols. 1-18, 20, 21. Madrid, España. Real Jardín Botánico, CSIC.

Domínguez Lozano, F., Galicia Herbada, D., Moreno Rivero, L. \& Moreno Sáiz, H. (1994). Asientos para un Atlas Corológico de la Flora Occidental. Mapa 663. Fontqueria 40, 200-201.

Fernández García-Rojo, C. (1998). Flora de la sierra de San Andrés y cuenca del Fresneda (Ciudad Real). Estudio de fronteras biogeográficas. Tesis Doctoral. Universidad de Jaén. Publicaciones del Herbario JAEN. Jaén, España. 401 pp.

Fernández García-Rojo, C. (2015). Aportación a la flora vascular en el tramo oriental y en el este del tramo central de Sierra Morena y estribaciones (Ciudad Real, Centro-Sur de la península Ibérica). Años 1990-2015. Blancoana 24, 1-181. (http://sites.google.com/site/ digitblancoana/).

Fernández García-Rojo, C. \& Fernández López, C. (1992). Plantas de la sierra de San Andrés (Ciudad
Real). Blancoana 9, 21-23.

Fernández López, C., Carazo Montijano, M. M. \& Hervás Serrano, J. L. (2006). Materiales para la flora del Alto Guadalquivir (Centro Sur de la península Ibérica). Catálogo de Flora Vascular. Publicaciones del Herbario Jaén. Jaén, España. 334 pp. (https://sites. google.com/site/herbariojaen/publicaciones).

García Río, R. (1998). Doce plantas del conjunto montañoso de Sierra Madrona. Anales del Jardín Botánico de Madrid 56(2), 402-404.

García Río, R. (2006). Flora y vegetación de Sierra Madrona y Valle de Alcudia. Bases científicas para su conservación. Centro de Investigaciones Ambientales del Mediterráneo (CIAMED). 336 pp.

GBIF. (2019). Portal de Datos de Biodiversidad. Nodo Nacional de Información en Biodiversidad (http://www. gbif.es).

Jury, S. L. (2003). Torilis Adans. In G. Nieto Feliner, S. L. Jury \& A. Herrero (Eds.), Flora iberica vol. 10 (pp. 84-92). Madrid, España. Real Jardín Botánico, CSIC.

López González, G. (2013). Gagea Salisb. In E. Rico, M. B. Crespo, A. Quintanar, A. Herrero \& C. Aedo (Eds.), Flora iberica vol. 20 (pp. 22-74). Madrid, España. Real Jardín Botánico, CSIC.

Martín Blanco, C. J. \& Carrasco, M. A. (2005). Catálogo de la flora vascular de la provincia de Ciudad Real. Monografias de la AHIM, 1.581 pp.

Martínez Ortega, M. M. (2013). Simethis Kunth. In E. Rico, M. B. Crespo, A. Quintanar, A. Herrero \& C. Aedo (Eds.), Flora iberica vol. 20 (pp. 314-317). Madrid, España. Real Jardín Botánico, CSIC.

Montserrat, P. (1981). Gagea del herbario Jaca y otras novedades florísticas. Anales del Jardín Botánico de Madrid 37(2), 619-627.

Morales del Molino, C. \& Mateo, R. G. (2010). Simethis mattiazzi (Vandelli) Saccardo [(=S. planifolia (L.) Gren.], redescubierta para la flora de Castilla-La Mancha (España). Acta Botanica Malacitana 35, 128-130.

Rivas Goday, S., Monasterio Fernández, A. \& Fernández Galiano, E. (1954). Islas atlánticas en pleno dominio de la flora mediterránea (Provincia de Ciudad Real). Anales de la Real Academia de Farmacia 20(5), 405412.

Rivas Martínez, S. (2007). Mapa de series, geoseries y geopermaseries de vegetación de España. Parte 1. Itinera Geobotanica 17, 5-436.

Salazar, C., Estrada, M. C., Rivas Rangel, A., Lendínez, M. L. \& Tercero, A. (2019). Silene mariana Pau. In J. C. Moreno Saiz, J. M. Iriondo Alegría, F. Martínez García, J. Martínez Rodríguez \& C. Salazar (Eds.), Atlas y Libro Rojo de la Flora Vascular Amenazada de España. Adenda 2017. Madrid, España. Ministerio de Transición Ecológica-Sociedad Española de Biología de la Conservación de Plantas. 170 pp. (en prensa).

Sánchez Gómez, P. \& Alcaraz, F. (1993). Flora, vegetación y paisaje vegetal de las sierras de Segura Orientales. Instituto de Estudios Albacetenses. Serie 1-Estudios $n^{\circ}$ 69. Albacete, España.

Talavera, S. (1990). Silene L. In S. Castroviejo, M. Laínz, G. López González, P. Montserrat, F. Muñoz Garmendia, J. Paiva \& L. Villar (Eds.), Flora iberica vol. 2 (pp. 313-406). Madrid, España. Real Jardín Botánico, CSIC. 